\title{
Fully InTEgrated Three Dimensional Sound InTENSITy SENSOR
}

D.R. Yntema, R.J. Wiegerink, J.W. van Honschoten, M. Elwenspoek

Transducers Science and Technology group

MESA+ Institute for Nanotechnology, University of Twente, the Netherlands

\begin{abstract}
For the first time, a complete 3-dimensional sound intensity sensor - consisting of 4 particle velocity sensors and a pressure microphone - has been integrated on a single chip, providing the possibility to do nearly point measurements of acoustic particle velocity, sound pressure, and therefore sound intensity. Principally the sensor consists of two distinct designs; a pressure sensor and particle velocity sensors. In this paper the design of the sensor, fabrication and measurement results are discussed and compared with theoretical results.
\end{abstract}

\section{INTRODUCTION}

Acoustic sound fields consist of a combination of pressure change and particle velocity. While acoustic pressure is a scalar quantity, particle velocity has both magnitude and direction. Therefore, to measure the complete sound field a sensor is needed for pressure and particle velocity, therefore consisting of a pressure sensor and a 3dimensional particle velocity sensor. Ideally, these sensors should be located in a single point, hence the integration of all sensors on a single chip. Furthermore, a small sensor significantly simplifies measurement of the sound field close to small sound sources.

\section{SENSOR DESIGN}

A 3-D particle velocity sensor consisting of four two-wire particle velocity sensors [1] was presented at MEMS'06 [2]. Further optimization of the design resulted in enhancements in nonintrusiveness, implying that the sensor has less influence on the sound field. Principally the sensor is still consisting of four pairs of sensor wires. The sensor design is schematically shown in figure 1 . Sensors 1 to 4 are under an angle of 45 degrees with the silicon plane. In the final design the sensor is made more acoustically transparent to improve directionality.

The integrated microphone, marked with a "P" in figure 1, does not contain moving parts like a diaphragm, but instead an acoustic pressure to particle velocity converter is realized so that the sound pressure can be measured by a fifth particle velocity sensor. This allows a relatively easy integration of the two kinds of sensors on a single substrate with a robust fabrication process. The particle velocity sensor is positioned at the entrance of an acoustic 'back chamber' which has the shape of a small meandering tube. The transfer function is the inverse acoustic impedance of this back chamber, which can be described as a standing wave tube with damping, [3] and [4], superimposed on the transfer function of the particle velocity sensor.

At the entrance side of this sensor one of the two sensor wires is parallel with the die surface and therefore the sensor could still be sensitive to flows in the plane with the die. Therefore a small cap is placed on top of the sensor, providing a sort of "particle velocity screen", see figure 5 b.

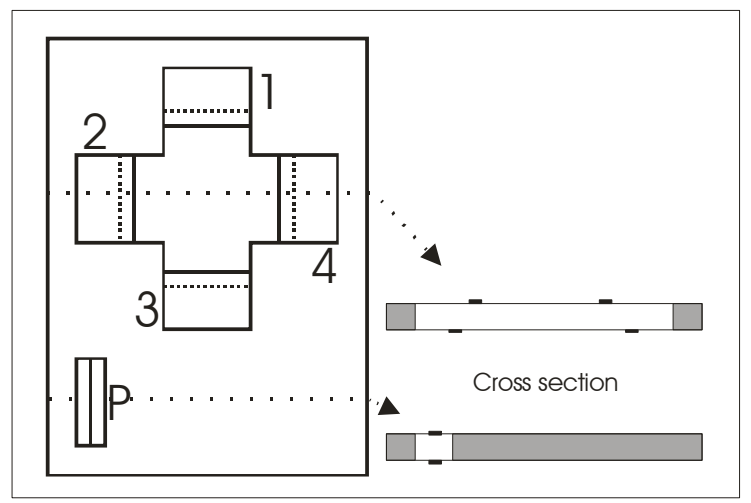

Figure 1; the sensor schematically depicted.

\section{THEORY OF THE PRESSURE SENSOR}

Imagine a small acoustically terminated tube with one open end. When now acoustic pressure in the vicinity of the tube opening increases there will be a particle velocity flow in the entrance of the tube pointed inwards due to pressure equalization of the tube. When a particle velocity sensor is placed in this inlet it will produce a signal dependent on pressure change, so a pressure to particle velocity transformer is made.

Acoustic impedance is defined as $z=p / u$. So when both the impedance and the pressure are given the particle velocity signal can be derived. As a result the acoustic impedance of the tube will reflect in the transfer function of the pressure to velocity device. The acoustic impedance of a terminated tube of length $x$ can be described as [3]:

$Z=\frac{1}{\rho c} \tanh (\Gamma k(l-x))$

With $\Gamma$ the reflection coefficient, $\rho$ the density of air, $c$ the speed of sound in air, $l$ the length of the tube, $k$ the wave number $\omega / c$, and $x$ the distance from the opening. The reflection coefficient can be described by different means; first of all it can be $i$, meaning that there is complete reflection and no 
damping inside the tube. This approximates measurement results roughly when using tubes with larger diameters (sizes in the order of centimeters and more combined with audio range frequencies). When using smaller tubes like in this sensor damping is of more importance. Damping in small tubes can be described with the Kirchhoff solution for damping, and secondly by the more complicated Low Reduced Frequency (LRD) model [4]. The latter model allows for non cylindrical tubes. The Kirchhoff solution uses for the damping coefficient $\Gamma=i+\frac{i+1}{\sqrt{2}} \frac{\lambda-1+\sigma}{s \sigma}$ with $s$ the sheer wave number, $\lambda$ the thermal conductivity of air and $\sigma$ the square root of the Prandtl number. The Kirchhoff model still gives a fair estimate of the damping inside a tube if the cross-sections are not too different. In figure 2 a comparison between both models is given.

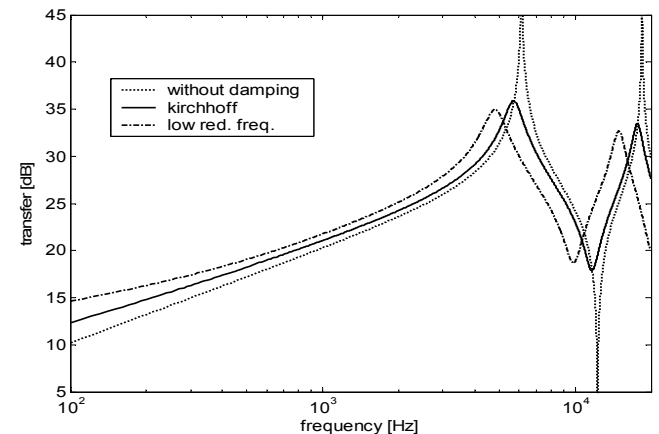

Figure 2; comparison between models.

The Kirchhoff model is evaluated with a circular tube with a diameter of $0.3 \mathrm{~mm}$, the low reduced frequency model with a tube with height $0.25 \mathrm{~mm}$ and width $1.5 \mathrm{~mm}$, which are the actual dimensions of the back chamber. Length of the tube is estimated to be $14 \mathrm{~mm}$ in both cases.

\section{FABRICATION}

Fabrication is done on both sides of a thin silicon wafer to be able to work "out of plane", which is necessary for wanted sensitivity directions. Double side polished $250 \mu \mathrm{m}$ thick wafers are used because the wafer thickness defines the distance between the sensor wires in this design. Platinum with thickness of $150 \mathrm{~nm}$ is deposited on a patterned silicon nitride surface of $200 \mathrm{~nm}$ on top of a silicon wafer. Next the photo resist is stripped away, leaving the patterned platinum. Following the silicon nitride is patterned for use as a $\mathrm{KOH}$ etching mask. This is done by RIE-etching holes in the silicon nitride. The places where silicon nitride should not be attacked are protected by photo resist. After $\mathrm{KOH}$ etching the sensor is ready, and can be broken out of the wafer by breaking the supports; see ' $A$ ' in figure 3 . The inner edges of the cross shape are attacked by the $\mathrm{KOH}$, but since sacrificial structures are placed (see
'B') there is sufficient silicon left over to avoid problems with this.

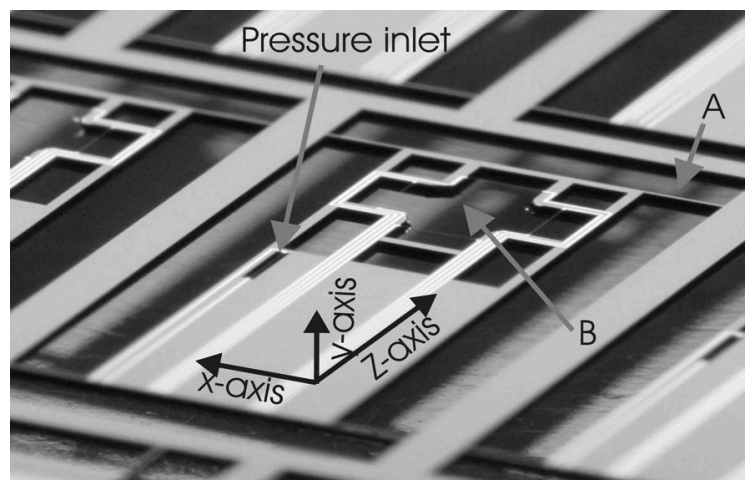

Figure 3; photo of the sensor on its wafer.
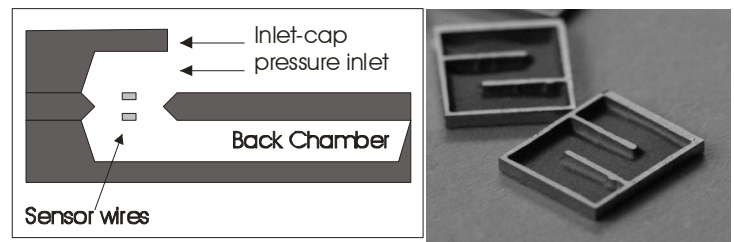

Figure 4a; the pressureflown schematically represented. Figure 4b); the back chamber.

For the pressure sensor the wires are placed on each side of the wafer, so the sensor wire-to-wire distance is $250 \mu \mathrm{m}$. This is shown in figure $4 \mathrm{a}$.

In a separate design the back chamber is made. This is done by patterning a silicon wafer with photo resist foil and powder blasting the cavity in silicon. After removing the remaining foil the chambers are diced. Both a back chamber and a pressure inlet are made using this method. The back chambers are glued manually to the die; however with some adjustments in the design the back chambers and inlet-caps can be etched in $\mathrm{KOH}$ and wafer-bonded to the sensor. Figure $4 \mathrm{~b}$ shows the back chamber, figure 5a shows the sensor chip with back chamber and figure $5 \mathrm{~b}$ shows the other side with the pressure inlet.

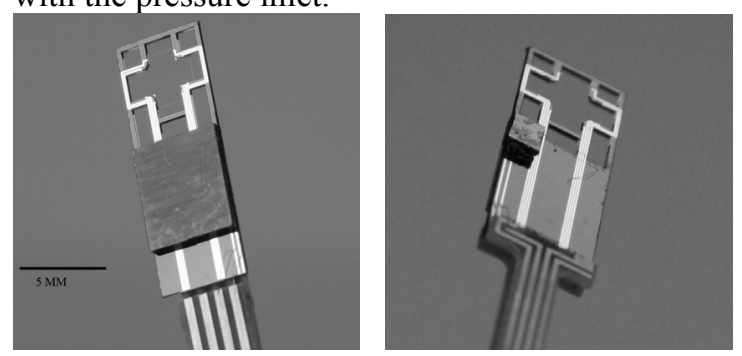

Figure 5; a) the pressure back chamber mounted, $5 b$; the inlet-cap mounted on the sensor die.

Figure 6 shows a summary of the fabrication process. For the particle velocity sensors the wanted directivity is 45 degrees out of plane, so here the sensor wires are displaced by $250 \mu \mathrm{m}$ in relation to each other. In this way the distance between the wires becomes $354 \mu \mathrm{m}$. 


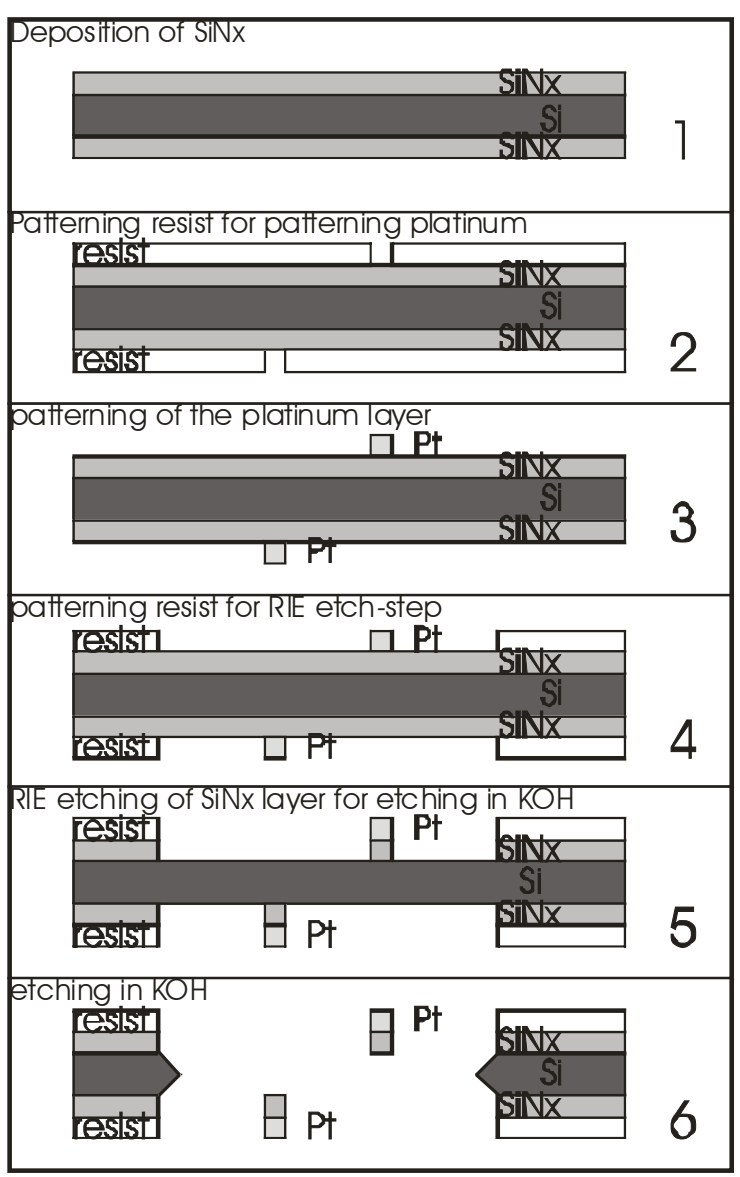

Figure 6; fabrication process in short.

\section{MEASUREMENT RESULTS}

For the pressure microphone the main goal is that it is indeed only sensitive to pressure. To confirm this the sensor was placed in a standing wave tube with a reference microphone at one end, the sensor at point ' $x$ ' and a loudspeaker generating noise at the other end. At certain frequencies there will be a maximum in particle velocity at point $\mathrm{x}$, and a minimum in pressure and vice versa. The reference microphone always measures a maximum in pressure because it is at the end of the tube. As a result, the measured transfer function between the sensor and the reference microphone contains peaks and zeros. Comparing the device with a standard pressure microphone, the peaks and zeros must be at the same frequencies. Comparing the device with a particle velocity sensor the peaks must exactly be at the dips of the particle velocity sensor. Figure 7 shows the result.

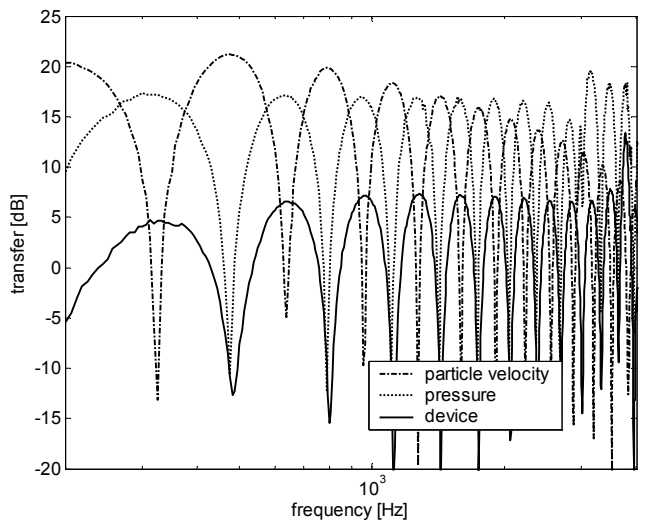

Figure 7; measurement proving pressure sensitivity.

For measurement of the response, the device is compared with a calibrated pressure microphone in a sound field. When looking at figure 2 the response of the back chamber clearly has a high pass behavior for frequencies below resonance. When this simulated behavior is compensated with the known low pass behavior of the microflown the theoretical results shown in figure 8 emerge. Now measurements can be compared with theoretic results. Both results together are shown in figure 8 .

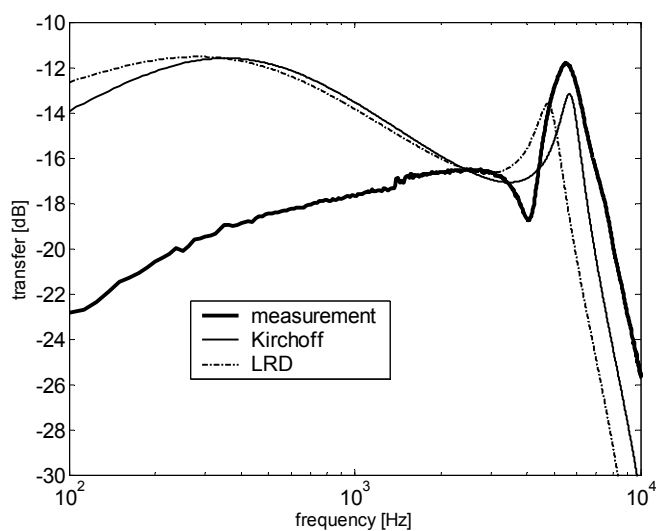

Figure 8; frequency response of the pressure device.

Clearly the response is a combination of the response of the microflown sensor and the pressure cavity. These effects combine to a more flat response.

A polar pattern is measured to prove that the pressure sensor is omni-directional. Again the sensor is placed inside a standing wave tube and the transfer function between the pressure microphone and the device is measured. This is done for 96 different angles. The results are plotted in figure 9. Clearly the sensor is omni directional. 

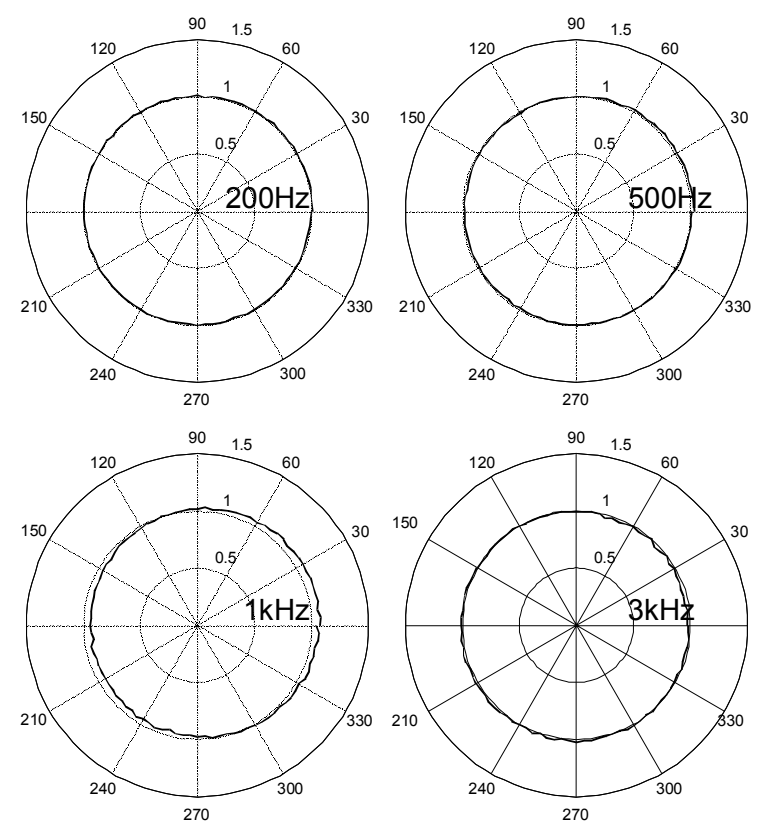

Figure 9; polar pattern of the pressure microphone, in linear scale.

For the 3D particle velocity sensors a polar pattern is measured and plotted in Figure 10a and b. The sensor is rotated inside a standing wave tube and the transfer function between the reference pressure sensor at one end and the device is measured. For 96 steps of rotation this is done and the result for $600 \mathrm{~Hz}$ is plotted. Polar patterns for the frequency range $100 \mathrm{~Hz}$ up to $5 \mathrm{kHz}$ (depending on the useable range of the standing wave tube) are verified and directivity does barely change in this frequency span. Clearly the polar pattern is a figure of eight, however the sensitivity direction differs approximately 15 degrees from the wanted direction of sensitivity. The deviation is constant and can be compensated for in a following design.

\section{CONCLUSION}

A fully integrated three dimensional sound intensity sensor on one die has been realized. Design and fabrication are discussed. The sensor consists of two distinct sensors; a pressure microphone and a particle velocity sensor. Both rely on the working principle of a particle velocity sensor. Theory involving the pressure sensor is compared with measurement results and pressure sensitivity of the sensor as well as its omni directionality is verified.

Measurements on the particle velocity sensor prove that the sensor is fully directional with a constant deviation for which can be compensated in a next design.

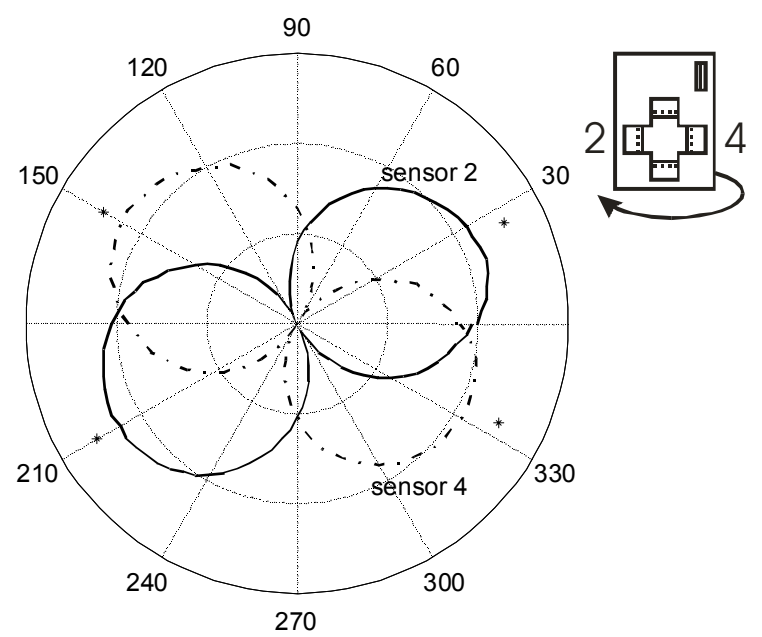

Figure 10a; polar pattern when rotated over Z-axis.

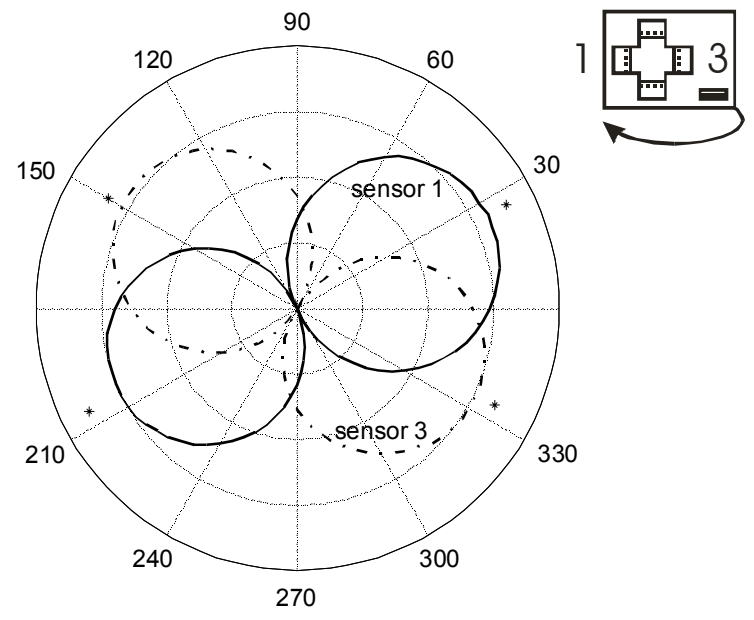

Figure 10b; polar pattern when rotated over X-axis.

\section{REFERENCES}

[1] H.-E. de Bree, "The Microflown: An acoustic particle velocity sensor," Acoustics Australia 31, 91-94 (2003).

[2] D.R. Yntema, J.W. van Honschoten, H.-E. de Bree, R.J. Wiegerink, M. Elwenspoek, "a three dimensional Microflown”, MEMS’06,

[3] D.R.Yntema, H-E deBree, "A Microflown based sound pressure microphone suitable for harsh environments", Noise-Con Minneapolis. october 17-19 2005.

[4] Frits v. Eerden, "Noise reduction with prismatic coupled tubes" ( $\mathrm{PhD}$ thesis), University of Twente, Enschede, The Netherlands, ISBN 9036515211 